\title{
Modelagem Matemática e Educação Ambiental no contexto da Cultura Digital
}

Arlindo José de Souza Júnior

Deive Barbosa Alves

\section{Resumo}

Neste artigo, analisamos o desenvolvimento de um projeto de educação ambiental relacionado a práticas de preservação da água. Este trabalho coletivo foi implantado com um grupo de alunos do $2^{\circ}$ ano do Curso Técnico de Meio Ambiente, integrado ao Ensino Médio Regular, de uma escola da Rede Federal na cidade de Uberlândia (MG). O objetivo do projeto foi o de incentivar os alunos a produzir ciência e tecnologia por meio da elaboração de protótipos digitais. O processo foi desenvolvido a partir da seguinte questão: Como a produção de uma tecnologia (descarga digital) possibilita a economia de água utilizada no vaso sanitário das nossas residências? A pesquisa prosseguiu de duas maneiras: levantamento sobre os dispositivos utilizados em um modelo comum de banheiro brasileiro e a construção de um protótipo com componentes digitais para o controle do volume de saída de água. Compreendemos que, além dos benefícios da busca pela preservação dos recursos naturais, este projeto contribuiu para a constituição do conhecimento derivado da modelagem matemática e seu vínculo com a cultura digital na educação, permitindo superar os obstáculos inerentes ao processo de construção desse conhecimento, bem como acelerar o processo de apropriação desses conhecimentos.

Palavras-chave: Cultura digital; Modelagem Matemática; Educação Matemática, Ensino Médio. 


\title{
Mathematical Modelling and Environmental Education in the context of Digital Culture
}

\author{
Arlindo José de Souza Júnior \\ Deive Barbosa Alves
}

\begin{abstract}
In this article, we analyze the development of an environmental education project related to water preservation practices. This collective work was implemented with a group of students of the 2nd year of the Technical Course of the Environment, integrated to the Regular High School, of a school of the Federal Network in the city of Uberlândia (MG). The purpose of the project was to encourage students to produce science and technology by developing digital prototypes. This process was developed from the formulation of the following question: How does the production of a technology (digital flush) enable the saving of water used in the toilet of our homes? The research proceeded in two ways: survey of the devices used in a common model of Brazilian bathroom and the construction of a prototype with digital components to control the volume of water output. We understand that, in addition to the benefits of the pursuit of preservation of natural resources, this project contributed to the constitution of knowledge derived from mathematical modeling and its link with digital culture in education, allowing to overcome the obstacles inherent in the process of building this knowledge, as well as accelerate the process of appropriation of this knowledge.
\end{abstract}

Keywords: Digital Culture; Mathematical modeling; Mathematical education, High school. 


\section{Introdução}

Uma escola pública da rede federal, no município de Uberlândia/MG, abriu espaço para a organização e desenvolvimento de um trabalho coletivo com educadores interessados na implantação de uma prática educativa que possibilitasse um diálogo com a matemática e as questões relacionadas ao meio ambiente. Segundo Freire,

[...] o fundamental, porém, é que a informação seja sempre precedida e associada à problematização do objeto em torno de cujo conhecimento ele dá esta ou aquela informação. Desta forma, se alcança uma síntese entre o conhecimento do educando, menos sistematizado - síntese que se faz através do diálogo (FREIRE, 2002, p. 65).

O objetivo do projeto foi incentivar estudantes que cursavam o $1^{\mathrm{o}}$ ano do Curso Técnico em Meio Ambiente Integrado ao Ensino Médio Regular, a interagirem com a matemática, no desenvolvimento de projetos relacionados à educação ambiental, pois

Certo conteúdo de matemática pode ser trabalhado por meio de um projeto de trabalho ou por meio de modelagem. A modelagem pode ser feita por meio de um projeto de trabalho se a obtenção do modelo matemático for o objetivo maior do trabalho, mas também pode ser apenas uma das etapas do projeto de trabalho se este for concebido como uma atividade que queira obter outros produtos que não sejam exclusivamente o modelo matemático (RIPARDO; OLIVEIRA; SILVA, 2009, p. 107).

A escola oferece o curso técnico em meio ambiente desde o ano de 2002. Mas, a partir do ano de 2013, o curso passou a ser ofertado na modalidade integrada ao Ensino Médio, destinado a alunos que concluíram o $9^{\circ}$ ano do ensino fundamental. Ela tem uma boa infraestrutura para o atendimento das necessidades técnicas e pedagógicas do curso técnico em meio ambiente: dispõe de laboratórios de microbiologia e de análise físico-química de água e de efluentes, além de estação climatológica, estação de tratamento de efluentes e viveiro de mudas nativas do cerrado (IFTM, 2015).

Neste artigo, a modelagem matemática foi desenvolvida com a utilização de Tecnologias da Informação e Comunicação - TIC. Segundo Skovsmose (2015, p. 16), "criar uma harmonia entre o trabalho de projecto e as actividades da sala de aula tem sido o grande desafio para a educação matemática baseada em projectos”. Ainda segundo esse pesquisador, "os computadores na educação matemática têm ajudado a estabelecer novos cenários para investigação" (SKOVSMOSE, 2015, p. 17).

Esse processo de interação, modelagem e TIC, é o que O’Sullivan e Igoe (2004) chamam de Physical Computing (Computação Física, em uma tradução literal). Segundo Igoe (2004), os sujeitos que trabalham com a Computação Física precisam, inicialmente, assistir as pessoas no cotidiano para detectar e interpretar suas interações. Interações que na Educação Ambiental para Meyer, Caldeira e Malheiros (2011, p. 100), o “[...] confere à aprendizagem e ISSN 2526-2882 
ao ensino a urgência do dia de hoje, da educação para o presente”. Caldeira e Meyer (2001), ao analisarem uma proposta de formação continuada de professores, destacam a importância da formulação de questões envolvendo a educação ambiental no processo de modelagem matemática.

Embora haja muitas definições da dinâmica a que se dá o nome de modelagem matemática, praticamente todas elas incluem a formulação da questão, em que a postura crítica se revela no instante em que se selecionam os aspectos essenciais de cada problema, para incluí-los no modelo matemático (tendo-se em mente que a tal escolha dos aspectos poderá, ou deverá ser alterada...). Esta formulação inclui tanto o estabelecer a questão em si quanto apresentar sua expressão numa linguagem do universo matemático, isto é, o problema matemático (CALDEIRA; MEYER, 2001, p. 157).

Nesta investigação discutimos o desafio coletivo de implantar uma prática educativa nas aulas de matemática relacionadas à formulação de problemas da Educação ambiental no contexto da cultura digital. Entendemos que o trabalho coletivo, além de possibilitar a produção de saberes necessários para o desenvolvimento do ensino com pesquisa, possibilita também o desenvolvimento de um ambiente favorável ao enfrentamento de diferentes tipos de desafios presentes no cotidiano escolar.

\section{O processo de produção da Descarga Digital}

Esse momento remete-nos à discussão sobre a ligação entre a 'modelagem matemática' e a 'cultura digital' na Educação Matemática. Compreendemos essa ligação como uma intersecção de quatro modos de produzir práticas e saberes: a matemática, a matemática aplicada, educação matemática e a cultura digital²0. Para Meyer, Caldeira e Malheiros (2011, p. 35), o que nós chamamos de matemática é o "conhecimento matemático produzido nas academias visando exclusivamente ao desenvolvimento da Matemática”.

A matemática aplicada, no entanto, "estuda e aprende Matemática para resolver algo" (Ibidem, p. 39). Já na educação matemática, há o acréscimo do aluno, variável que não se apresenta nos dois anteriores. Por conseguinte, faz-se necessário agir e refletir no sentido de educar matematicamente um interlocutor. Mas a afirmativa especifica que esse, o interlocutor, se produz e produz em uma cultura digital, a qual o conhecimento nessa cultura, segundo Deuze (2006), dá-se pelo entrelaçamento remixado ${ }^{21}$ entre tecnologias antigas e novas com uma contínua, personalizada e mais ou menos autônoma montagem, desmontagem e

\footnotetext{
${ }^{20}$ Nesse artigo, cultura digital pode ser compreendida como um conjunto emergente de valores, práticas e expectativas em relação à forma como as pessoas agem e interagem em uma sociedade contemporânea em rede.

${ }^{21}$ Obra modificada por outra pessoa ou pelo próprio autor. 
remontagem da realidade mediada. Esse contexto é intrinsecamente ligado ao questionamento: qual a utilidade da matemática para os alunos?

[...] a Matemática serve para que a gente possa fazer uso dela, e, a partir desse uso, compreender mais da realidade, compreender mais das situações da vida. E acreditarmos que, para os alunos...Desta maneira, quando deslocamos essa ideia da Matemática Aplicada, sustentada pela Matemática Pura, para as questões educacionais, deve sempre existir a consciência de que há ali alunos que precisam aprender Matemática para viver, e é necessário saber o que esse aluno precisa saber de Matemática, para que precisará dela e como essa Matemática vai chegar até ele (MEYER; CALDEIRA; MALHEIROS, 2011, p. 39, grifos nossos).

Por esses dizeres se busca, então, o aprendizado matemático crítico e comprometido com os problemas do mundo real, com a matemática e com o social. Esse aprender se procede pela modelagem matemática, por ter como característica quatro passos, três tradicionais dela e um advindo da cultura digital: "o da formulação, o do estudo de resolução (ou, em muitos casos - aliás a maioria - o de resolução aproximada), o de avaliação" (MEYER; CALDEIRA; MALHEIROS, 2011, p. 17, grifos do autor), e o remix que, aqui, podemos chamar de protótipo, pois esse representa um primeiro modelo (produto) modificado por outra pessoa ou pelo próprio produtor, emaranhado por tecnologias antigas e novas. Contudo, segundo Biembengut (2014) é necessário, nesse processo, que é investigativo, discutir e refletir tanto da avaliação ou validação do modelo matemático encontrado quanto da aprendizagem que processo proporcionou ao grupo. Sobre modelo matemático Stewart (2015, p. 22) ressalta que:

Um modelo matemático nunca é uma representação completamente precisa de uma situação física - é uma idealização. Um bom modelo simplifica a realidade o bastante para permitir cálculos matemáticos, mantendo, porém, precisão suficiente para conclusões significativas. É importante entender as limitações do modelo. A palavra final está com a Mãe Natureza.

A partir desse referencial teórico o grupo "Natureza Blue" adaptou, dos escritos de Meyer, Caldeira e Malheiros (2011), Biembengut (2014) e Deuze (2006), propondo fases cíclicas do processo de Modelagem Matemática: na primeira fase formulamos um problema; na segunda buscamos informações, planejamos e executamos prováveis soluções, construção do modelo matemático; na terceira criamos protótipos, jogo ou robô, é o remix; na quarta etapa discutimos sobre a relação entre o produto e o real; por fim, na quinta fase refletimos sobre o que aprendemos durante o processo. A Figura 1 - Ilustração das fases da Modelagem sintetiza tais dizeres. 
Figura 1 - Ilustração das fases da Modelagem

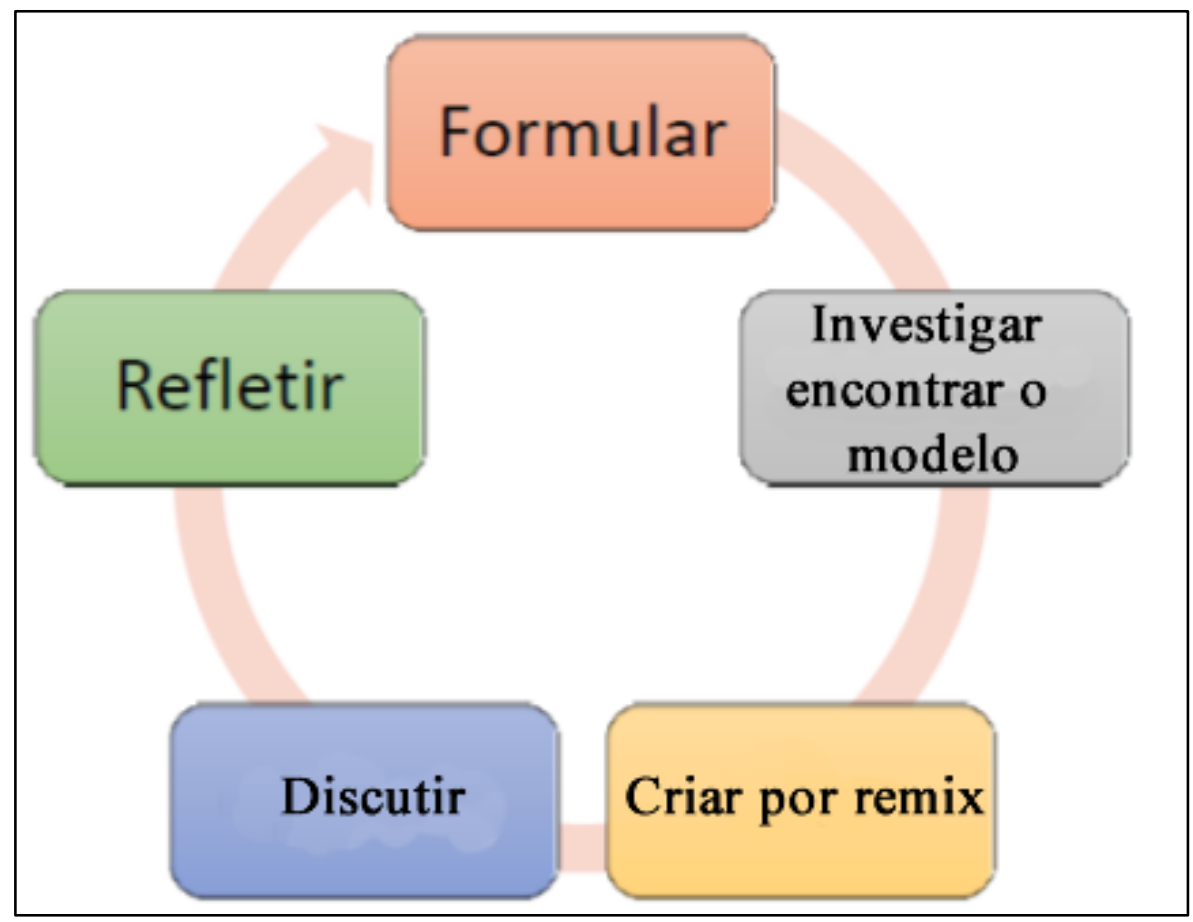

Fonte: Adaptado de Meyer, Caldeira e Malheiros (2011), Biembengut (2014) e Deuze (2006)

Seguir esse caminho teve como objetivo propiciar ao grupo "Natureza Blue" sua inserção inicial à produção investigativa de prática e saberes matemáticos, concomitante à consciência da relevância de se usar esses saberes em uma cultura digital para a melhoria da vida. Isso se deu envolvendo de forma direta três disciplinas: Matemática, Introdução à Metodologia Científica e Gestão Ambiental. Usou-se a Matemática, Meio Ambiente e Tecnologia como tema estruturador dos projetos de trabalho. Os projetos de trabalho, Alves (2017), foram pautados pela construção coletiva do conhecimento, pela interação entre os diferentes saberes e olhares e pelo esforço conjunto na resolução de problemas práticos.

As etapas ilustradas na Figura 1 foi aplicada a turma de 2015 do ensino técnico em Meio Ambiente do IFTM que tinha 35 alunos. Educandos que no processo de produção de trabalho com projetos foram divididos em grupos de três a cinco sujeitos. Para este trabalho foi escolhido a produção de um grupo que se intitulou de "Natureza Blue", esforços de três alunas que queriam participar da $12^{\mathrm{a}}$ edição da Semana Nacional de Ciência e Tecnologia (SNCT), a qual em 2015 teve como tema "Luz, ciência e vida”. Esse tema foi baseado em decisão da Assembleia Geral das Nações Unidas, que proclamou o referido ano como "Ano Internacional da Luz, com o objetivo de celebrar a luz como matéria da ciência e do desenvolvimento tecnológico" (MCTIC, 2015, p. 1). Elas almejavam criar um dispositivo tecnológico que com pouca energia elétrica que evitasse o desperdício de água em residências. As alunas narram a formação desse grupo, da seguinte forma: 
Somos três amigas ambientalistas que já se conhecem desde o início da vida no IF e juntamente com os professores iremos desenvolver o tema "Economia de Água" [...] formamos o grupo "Natureza Azul". Somos estudantes (e amigas!) do $2^{\circ}$ ano do Curso Técnico em Meio Ambiente do Instituto Federal de Educação, Ciência e Tecnologia do Triângulo Mineiro (IFTM) [...] (Documento produzido na pesquisa).

Esse grupo iniciou o desenvolvimento do projeto pela familiarização da situaçãoproblema. Uma aluna apresentou a todos nós informações do jornal Gazeta on-line do Espírito Santo, as quais discorriam sobre o desperdício de água em casa: "o banheiro é o campeão em desperdício nas residências, já que representa $60 \%$ de todo consumo de água” (GAZETA, 2010, p. 1). E o "grande" culpado é o vaso sanitário. Assim, ficou decidido que o grupo "Natureza Blue" investigaria possibilidades de economia de água no vaso sanitário.

O reconhecimento dessa situação-problema teve, também, a leitura da norma brasileira (NBR) 15.097/04 estabelecida pela Associação Brasileira de Normas Técnicas (ABNT), a qual estabelece que qualquer descarga - independente do modelo ou tipo - deve consumir no máximo 6 litros de água (ABNT, 2004; GAZETA, 2010). Outra informação foi obtida do Instituto Nacional de Metrologia, Qualidade e Tecnologia (Inmetro), o mesmo afirma que há "3 (três) principais mecanismos de sistemas de descarga disponíveis no mercado de consumo, a saber, válvulas de descarga, caixa de descarga não acoplada e caixa de descarga acoplada” (INMETRO, 2014, p. 4). Assim, para simplificar a situação-problema o grupo escolheu trabalhar com caixa de descarga acoplada, nesse sistema de vaso sanitário "a caixa é acoplada à parte traseira da bacia, o que requer uma bacia específica. As caixas e bacias, neste caso, são comercializadas em conjunto" (INMETRO, 2014, p. 4).

Depois dessa familiarização o grupo, no processo dialógico de investigações, propôs o seguinte problema: será que a produção de uma tecnologia (uma descarga digital), que possibilita digitar a quantidade de litros d'água a ser usada no vaso sanitário, favorece a economia de água em uma residência?

A partir do problema formulado o grupo optou por fazer uma caixa acoplada de vidro com o "Reparo Completo Universal Acionador Superior Caixa Acoplada”. Nesse conjunto tem: mecanismo de entrada, mecanismo de saída e botão superior. A Figura 2 - Caixa de Vidro e conjunto de reparo universal mostra o conjunto de reparo e caixa de vidro. 
Figura 2 - Caixa de Vidro e conjunto de reparo universal

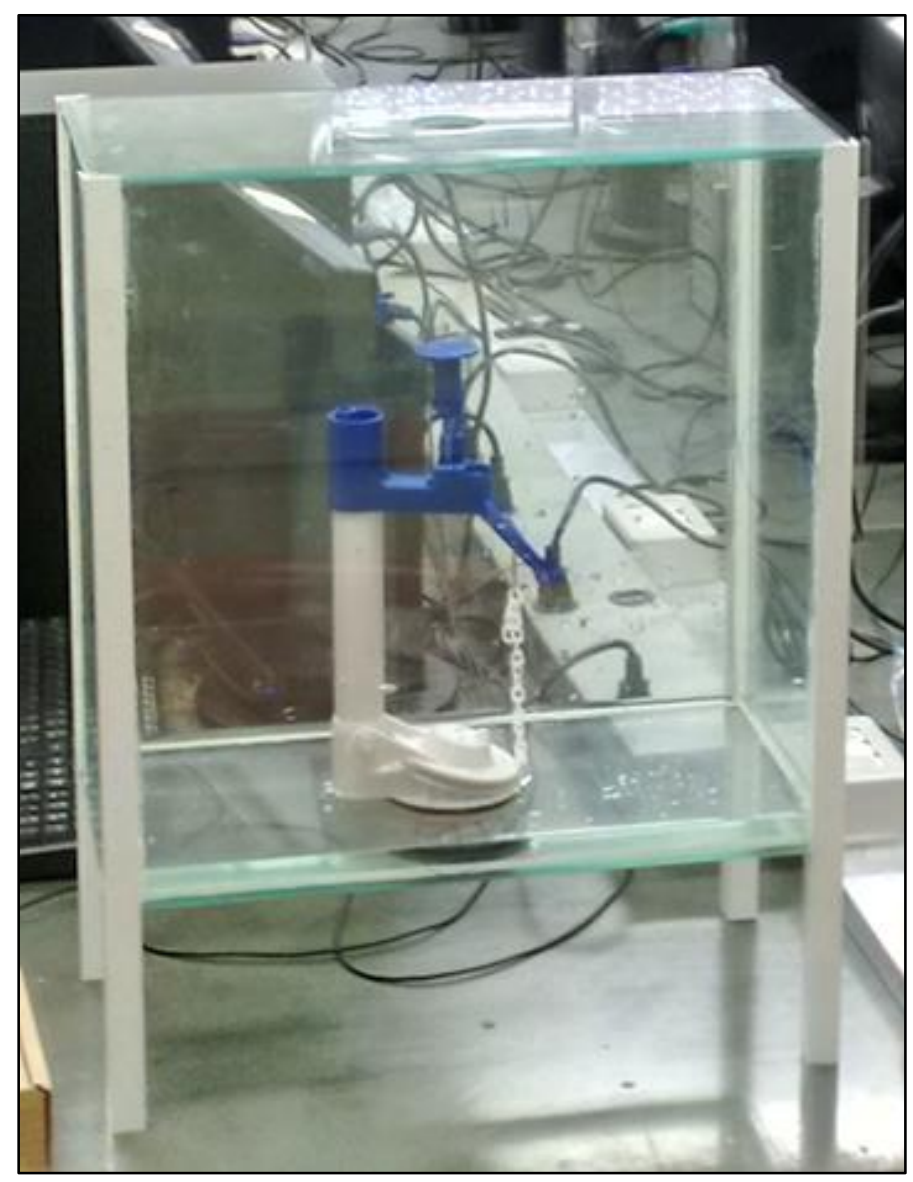

Fonte: Alves (2017)

No estudo desprezou-se os usuários de caixa acoplada com um botão de acionamento dual. As alunas passaram a investigação, matematicamente, a caixa acoplada calculando o volume de uma caixa acoplada comum, a qual representamos, com suas respectivas medidas, na Figura 3 - Medidas da caixa acoplada de um vaso sanitário.

Figura 3 - Medidas da caixa acoplada de um vaso sanitário

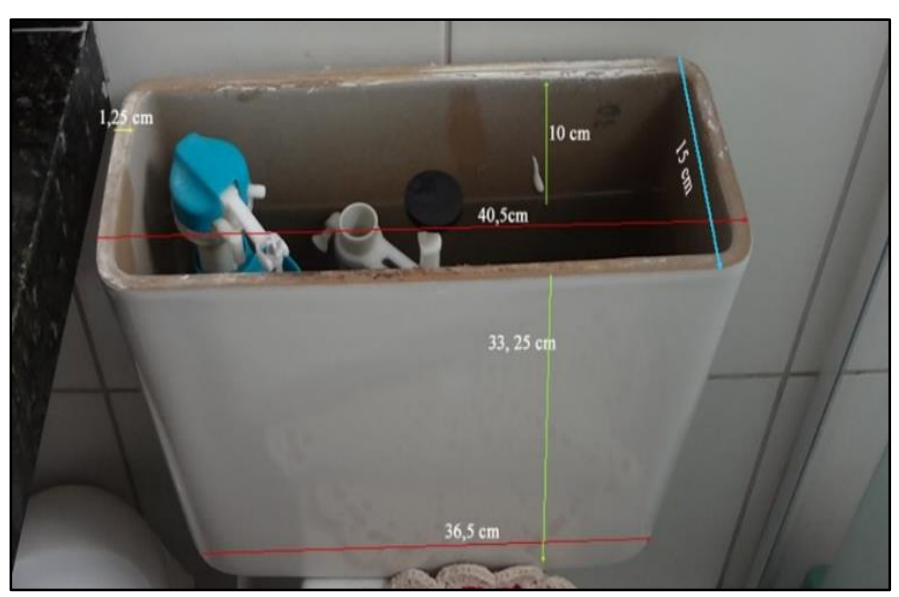

Fonte: Alves (2017)

ISSN 2526-2882

$* 157 *$ 
Para calcularem o volume de água na caixa acoplada foi atribuir às imagens formas geométricas conhecidas. Atribuíram à caixa o modelo geométrico de um tronco de pirâmide retangular. Segundo Smole e Diniz (2013), define-se pirâmide da seguinte forma: "Seja uma superfície poligonal R contida em um plano a e um ponto V fora de $\alpha$ (veja Figura 4 - Definição de pirâmide). A região de todos os segmentos de reta com uma extremidade em $\mathrm{V}$ e a outra em R é denominada pirâmide” (SMOLE E DINIZ, 2013, p. 157, grifos das autoras).

Figura 4 - Definição de pirâmide

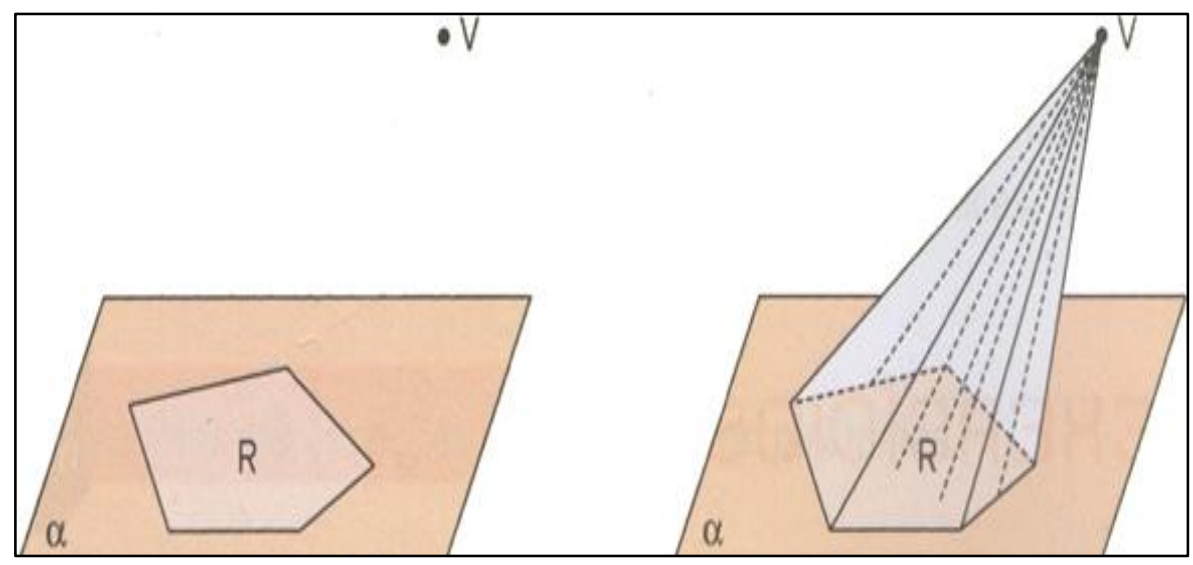

Fonte: adaptado de Smole e Diniz (2013)

Esse modelo geométrico foi atribuído à caixa acoplada, especificando na pirâmide, de base retangular, o tronco dela, que representa a própria caixa e outro tronco, que representa a água que está contida na caixa acoplada. A Figura 5 - Modelo geométrico da caixa acoplada, medidas em centímetro $(\mathrm{cm})$ representa esses dizeres.

Figura 5 - Modelo geométrico da caixa acoplada, medidas em centímetro $(\mathrm{cm})$

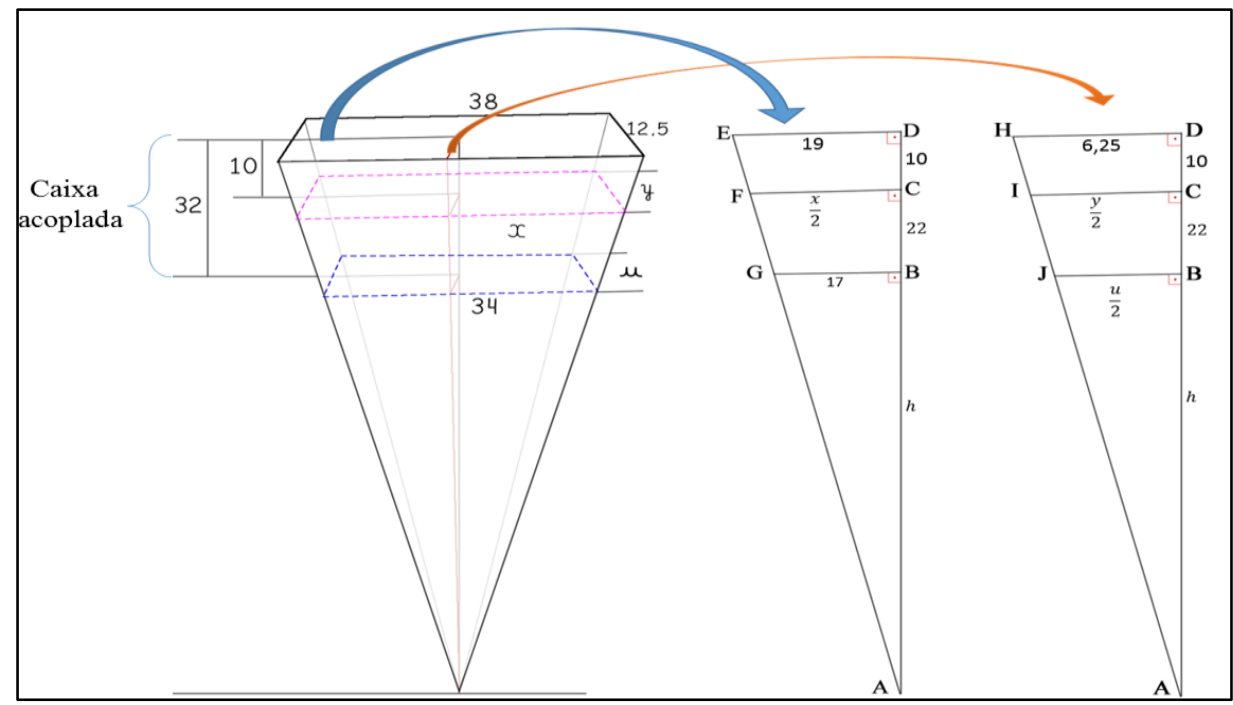

Fonte: Alves (2017) 
Para encontrar o volume correto, foi necessário encontrar as dimensões dos retângulos tracejados, ou seja, os valores de x, y e u da Figura 5- Modelo geométrico da caixa acoplada, medidas em centímetro $(\mathrm{cm})$. Logo, precisamos perceber os triângulos formados: ABG, ACF e ADE, da Figura, e os triângulos: ABJ, ACI e ADH. Note que todos são triângulos retângulos e semelhantes entre si, pelo Caso AA - Ângulo-Ângulo: "Se dois triângulos possuem dois ângulos ordenadamente congruentes, então eles são semelhantes” (UFRGS, 2016, p. 1). Podemos observar, pela figura, que todos os triângulos têm o mesmo ângulo Â e também o de $90^{\circ}$. Desse ponto de vista, da semelhança entre os triângulos $A \widehat{B} G$ e $A \widehat{D} E$, os seguintes segmentos são proporcionais:

$$
\frac{\overline{D E}}{\overline{B G}}=\frac{\overline{A D}}{\overline{A B}} \leftrightarrow \frac{19}{17}=\frac{h+32}{h} \leftrightarrow 19 \cdot h=17 \cdot(h+32) \leftrightarrow 2 \cdot h=544 \leftrightarrow h=\frac{544}{2}=272 \mathrm{~cm}
$$

Agora, pela semelhança entre os triângulos $A \widehat{B} G$ e AĈF, temos essa outra proporcionalidade:

$$
\frac{\overline{C F}}{\overline{B G}}=\frac{\overline{A C}}{\overline{A B}} \leftrightarrow \frac{\frac{x}{2}}{17}=\frac{h+22}{h} \leftrightarrow 136 \cdot x=4998 \leftrightarrow x=\frac{4998}{136} \leftrightarrow \mathrm{x}=36,75 \mathrm{~cm}
$$

A partir da Figura 5 encontramos os valores de $h=272 \mathrm{~cm} \mathrm{e} \mathrm{x}=36,75 \mathrm{~cm}$, agora, para finalizar, temos que calcular o valor do $y e$ de $u$. Esses valores podem ser encontrados observando a Figura 5, pois é dela que vemos a semelhança entre os triângulos AĈI e A $\widehat{D} H$ que garantem a proporcionalidade entre os seguintes segmentos:

$$
\frac{\overline{C I}}{\overline{D H}}=\frac{\overline{A C}}{\overline{A D}} \leftrightarrow \frac{\frac{y}{2}}{6,25}=\frac{294}{304} \leftrightarrow 152 \cdot y=1837,5 \leftrightarrow y=\frac{1837,5}{152} \leftrightarrow y \approx 12 \mathrm{~cm}
$$

Por fim, para encontrar o valor de $\mathrm{u}$, basta trabalharmos com os triângulos semelhantes $A \widehat{B}$ J e $A \widehat{D} H$, uma vez que asseguram a proporcionalidade entre os segmentos:

$$
\frac{\overline{B J}}{\overline{D H}}=\frac{\overline{A B}}{\overline{A D}} \leftrightarrow \frac{\frac{u}{2}}{6,25}=\frac{272}{304} \leftrightarrow 152 \cdot u=1700 \leftrightarrow u=\frac{1700}{152} \leftrightarrow u \approx 11 \mathrm{~cm}
$$

Podemos, com esses valores calculados, representá-los na pirâmide retangular que mostramos na Figura 6 - Pirâmide retangular da caixa acoplada com medidas em centímetro (cm). A partir das medidas nela apresentadas, podemos calcular o volume da caixa acoplada. 
Nessa figura observamos que há duas pirâmides: ABCDE e AFGHI. A diferença do volume dessa com aquela nos dá o volume de água da caixa acoplada.

Figura 6 - Pirâmide retangular da caixa acoplada com medidas em centímetro (cm)

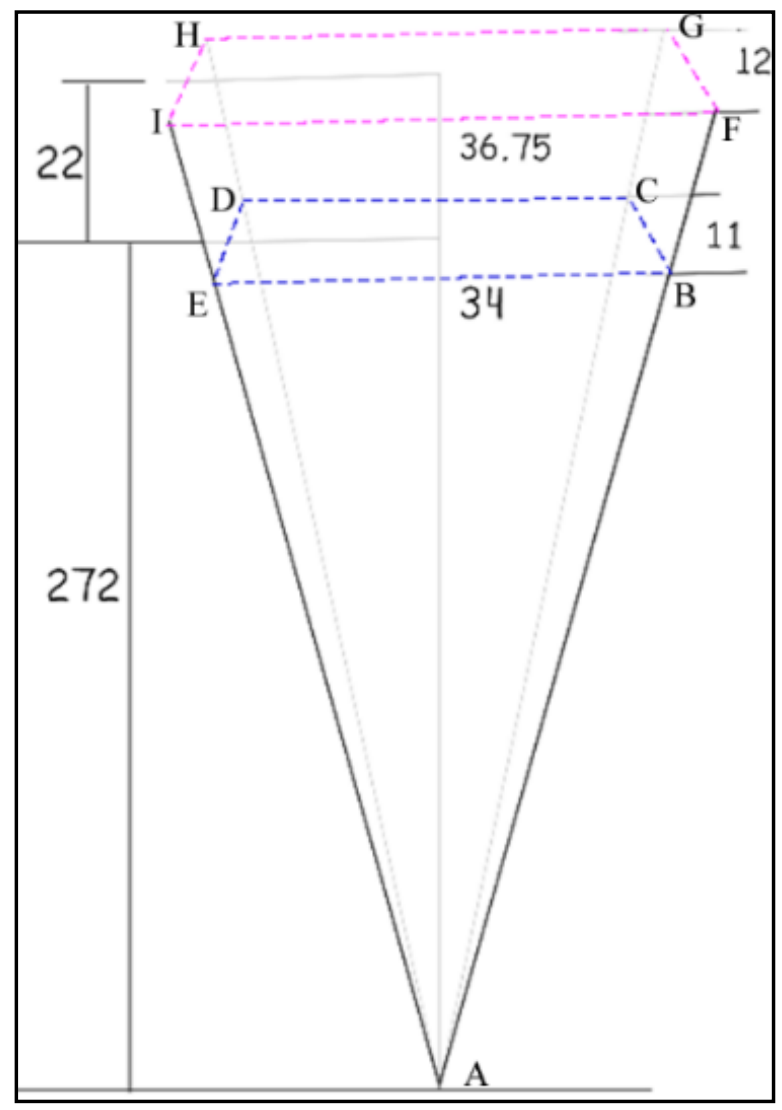

Fonte: Alves (2017)

Segundo Smole e Diniz (2013, p. 204), o "volume de qualquer pirâmide é $\frac{1}{3}$ do produto da área da sua base pela medida da sua altura”, ou seja:

$$
\mathrm{V}=\frac{1}{3} \cdot \mathrm{A}_{\mathrm{b}} \cdot \mathrm{h}
$$

Em que $A_{b}$ é a área da base e h é a altura da pirâmide. Vamos calcular em primeiro lugar o volume da pirâmide AFGHI, pela Figura 6 - Pirâmide retangular da caixa acoplada com medidas em centímetro $(\mathrm{cm})$ temos que $\mathrm{h}=294$ e $\mathrm{A}_{\mathrm{b}}=36,75 \cdot 12=441 \mathrm{~cm}^{2}$, dessa forma, $\mathrm{o}$ volume da pirâmide AFGHI é:

$$
\mathrm{V}_{\mathrm{AFGHI}}=\frac{1}{3} \cdot 441 \cdot 294=43218 \mathrm{~cm}^{3}
$$

O mesmo deve ser feito para a pirâmide $\mathrm{ABCDE}$, mas lembrando que, para ela, o valor de h é 272 e área da base é $A_{b}=34 \cdot 11=374 \mathrm{~cm}^{2}$, assim o volume da pirâmide ABCDE é: 


$$
\mathrm{V}_{\mathrm{ABCDE}}=\frac{1}{3} \cdot 374 \cdot 272 \approx 33909 \mathrm{~cm}^{3}
$$

Com o volume das duas pirâmides calculado pode-se encontrar uma aproximação para o valor do volume de água aparente ( $V_{\text {água aparente }}$ ) da caixa acoplada, da seguinte forma:

$$
\mathrm{V}_{\text {água aparente }} \approx \mathrm{V}_{\mathrm{AFGHI}}-\mathrm{V}_{\mathrm{ABCDE}} \approx 43218-33909 \approx 9309 \mathrm{~cm}^{3}
$$

Colocamos o termo "aparente", pois há dentro da caixa acoplada, os mecanismos de entrada e saída de água que adicionam volume aos $9309 \mathrm{~cm}^{3}$ encontrados. Embora existam diversos mecanismos, o que estamos considerando aqui é um kit de entrada MASTERFLUX mais saída convencional22, da marca Censi. A Figura 7 - Kit de reparo MASTERFLUX Censi para caixa acopladaapresenta seus componentes.

Figura 7 - Kit de reparo MASTERFLUX Censi para caixa acoplada

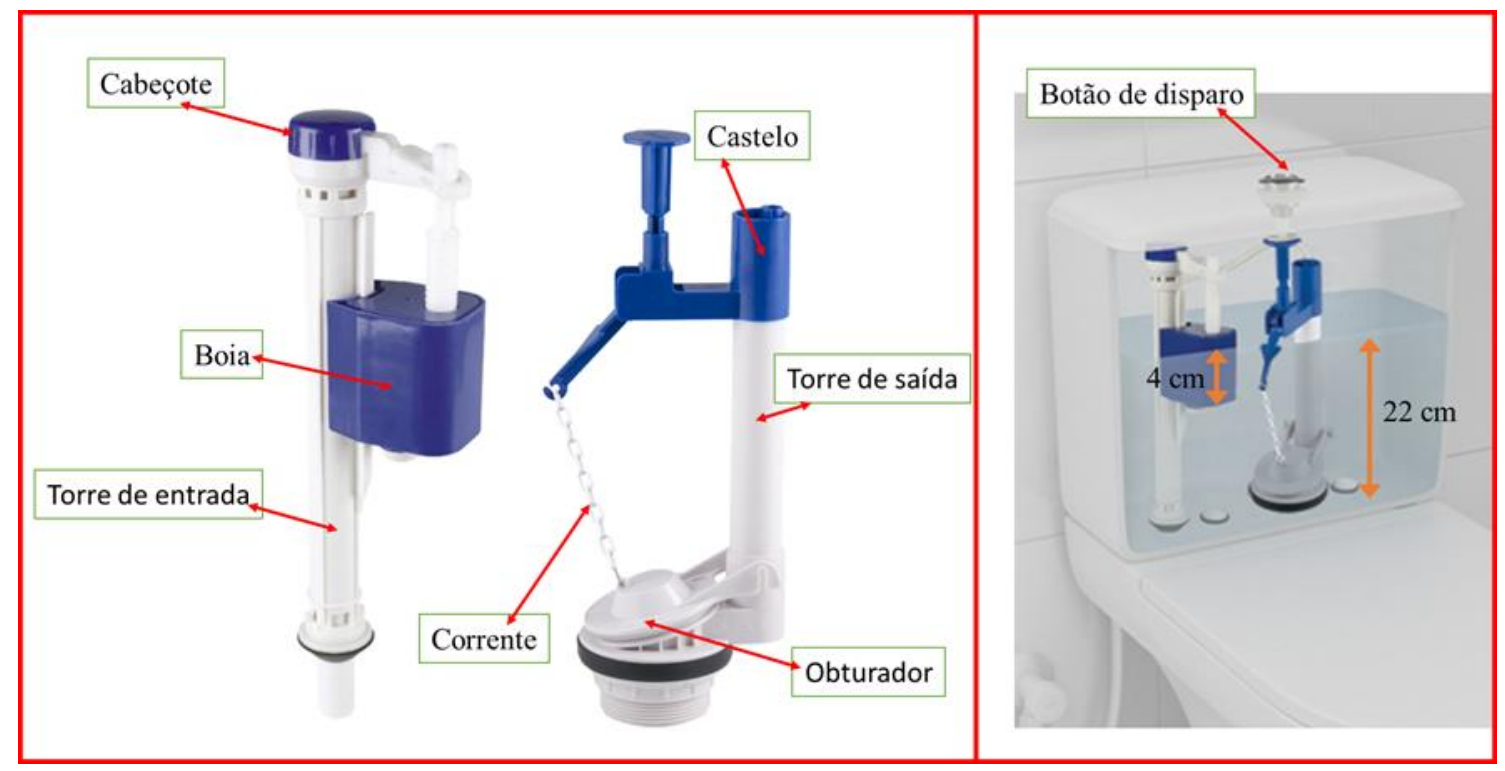

Fonte: adaptado de Censi (2016)

Como podemos observar na figura o kit é composto de mecanismo de entrada que é composto pelo: cabeçote, boia e torre de entrada. E por um mecanismo de saída que é composto pelo: castelo, torre de saída, obturador, a corrente e o botão de disparo. Na nossa caixa acoplada a torre de entrada e saída tinham o mesmo diâmetro, aproximadamente 2,7 centímetros, a boia tinha um comprimento de $5 \mathrm{~cm}$ e de largura $4 \mathrm{~cm}$. Já obturador tinha um diâmetro de 7,5 cm e uma espessura dentro da água de aproximadamente $0,5 \mathrm{~cm}$. Com tais medidas o grupo desenhou os modelos geométricos que representavam os mecanismos 
imersos na água da caixa acoplada. A Figura 8 - Modelo geométrico dos mecanismos da caixa acoplada apresenta tais modelos.

Figura 8 - Modelo geométrico dos mecanismos da caixa acoplada

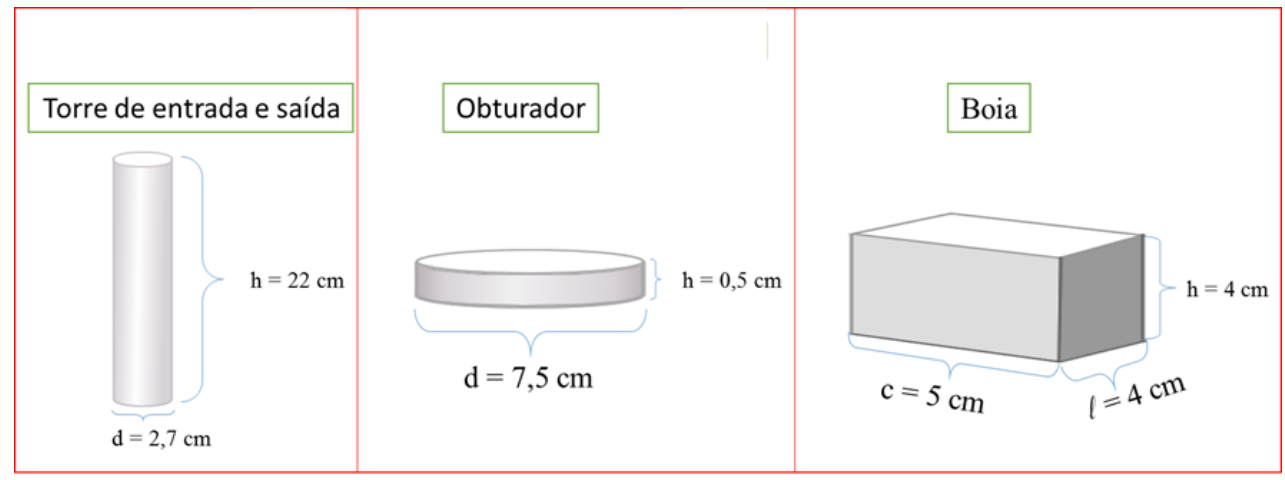

Fonte: Alves (2017)

Consideramos que a torre de entrada e saída o mesmo modelo geométrico, nesse caso, um cilindro. Segundo Smole e Diniz (2013) o volume de um cilindro circular pode ser encontrado pelo modelo matemático:

$$
\mathrm{V}=\pi \cdot r^{2} \cdot \mathrm{h}
$$

Em que $r$ é raio do círculo, h é a altura do cilindro e $\pi$ uma constante irracional que adotaremos o valor aproximado de 3,14. Assim, sabendo que $\mathrm{h}=22 \mathrm{~cm} \mathrm{e} \mathrm{r}=3,75 \mathrm{~cm}$, temos o volume das torres:

$$
\mathrm{V}_{\text {torres }}=3,14 \cdot(1,35)^{2} \cdot 22 \approx 126 \mathrm{~cm}^{3}
$$

Como são duas torres, então o volume de $252 \mathrm{~cm}^{3}$. Já o volume do obturador, é:

$$
\mathrm{V}_{\text {obturador }}=3,14 \cdot(3,75)^{2} \cdot 0,5 \approx 22 \mathrm{~cm}^{3}
$$

Por fim, o volume da boia é dado por um paralelepípedo reto - retângulo, que segundo Smole e Diniz (2013) pode ser calculado da seguinte forma: $V=A_{b} \cdot h$. Em que $A_{b}$ é área do retângulo que é a base e h é a altura do paralelepípedo. Nossa boia tem $\mathrm{h}=4 \mathrm{~cm} \mathrm{e} \mathrm{A}_{\mathrm{b}}=5 \cdot 4=$ $20 \mathrm{~cm}$, logo com esses valores temos o volume: $\mathrm{V}_{\text {boia }}=20 \cdot 4=80 \mathrm{~cm}^{3}$. Para sabermos o volume real de água $\left(\mathrm{V}_{\text {água }}\right)$ da caixa acoplada temos de retirar do volume de água aparente dos volumes: da boia, do obturador e das torres de entrada e saída. Assim, teremos volume real de água na caixa acoplada:

$$
\begin{gathered}
\mathrm{V}_{\mathrm{a} \text { gua }}=\mathrm{V}_{\text {água aparente }}-\mathrm{V}_{\text {boia }}-\mathrm{V}_{\text {obturador }}-\mathrm{V}_{\text {torres }} \\
\mathrm{V}_{\text {água }}=9309-80-22-252 \\
\mathrm{~V}_{\text {água }}=8955 \mathrm{~cm}^{3}
\end{gathered}
$$

Passamos esse valor para litros teremos aproximadamente 9 litros gastos com água para limpar a bacia do vaso sanitário. Sabendo do volume que nossa descarga gastava os alunos ISSN 2526-2882 
coletaram de seis pessoas o uso diário no mês de abril de 2015 dos dejetos sólidos e líquidos depositados no vaso sanitário. Dessa forma estabeleceu-se tanto o volume de água por descarga, $\mathrm{V}_{\text {água }}=9 l$, quanto ao uso dela, em média, por dia, os dejetos sólidos $\left(\overline{\mathrm{x}}_{S}\right)$ foi uma vez e o dejeto líquido $\left(\overline{\mathrm{x}}_{L}\right)$ foram seis vezes, ou seja, $\overline{\mathrm{x}}_{S}=1$ e $\overline{\mathrm{x}}_{L}=6$. A partir daí foi possível estabelecer o consumo médio diário de água usada para limpar a bacia sanitária, bem como a função de economia de água, considerando duas hipóteses:

1. Em dejetos sólidos se consome todo o volume de água da caixa acoplada;

2. Em dejetos líquidos reduzir o consumodo volume de água a um valor maior que zero e menor que o volume de água da caixa acoplada. Mas, uma vez escolhido o valor ficaria inalterado por trinta dias.

Por tais hipóteses simplificadoras, usando uma constante de economia $(e)$ na função de consumo de água para limpeza da bacia sanitária $(y(x))$, medidos em litros, pelos dias de uso $(\mathrm{x})$ foi possível chegar ao modelo geral:

$$
y(\mathrm{x})=\left(\mathrm{V}_{\text {água }} \cdot \overline{\mathrm{x}}_{S}+\left(\mathrm{V}_{\text {água }}-e\right) \cdot \overline{\mathrm{x}}_{L}\right) \cdot \mathrm{x}
$$

Quadro 1 - Modelos de economia de água

\begin{tabular}{|c|c|c|c|c|c|}
\hline $\mathbf{V}_{\text {água }}$ & $\overline{\mathbf{x}}_{S}$ & $\overline{\mathbf{x}}_{L}$ & $\boldsymbol{e}$ & $\boldsymbol{y}(\mathbf{x})=\left(\mathbf{V}_{\text {água }} \cdot \overline{\mathbf{x}}_{S}+\left(\mathbf{V}_{\text {água }}-\boldsymbol{e}\right) \cdot \overline{\mathbf{x}}_{L}\right) \cdot \mathbf{x}$ & Modelos \\
\hline 9 & 1 & 6 & $e=0$ & $y(x)=(9 \cdot 1+(9-0) \cdot 6) \cdot x$ & $\begin{array}{l}y(\mathrm{x})=63 \cdot \mathrm{x}, \text { em trinta dias, } \mathrm{x}= \\
\text { 30, gasta-se } 1890 \text { litros }\end{array}$ \\
\hline 9 & 1 & 6 & $e=1$ & $y(x)=(9 \cdot 1+(9-1) \cdot 6) \cdot x$ & $\begin{array}{l}y(\mathrm{x})=57 \cdot \mathrm{x}, \text { em trinta dias, } \mathrm{x}= \\
30, \text { gasta-se } 1710 \text { litros }\end{array}$ \\
\hline 9 & 1 & 6 & $e=2$ & $y(x)=(9 \cdot 1+(9-2) \cdot 6) \cdot x$ & $\begin{array}{l}y(\mathrm{x})=51 \cdot \mathrm{x}, \text { em trinta dias, } \mathrm{x}= \\
30, \text { gasta-se } 1530 \text { litros }\end{array}$ \\
\hline 9 & 1 & 6 & $e=3$ & $y(x)=(9 \cdot 1+(9-3) \cdot 6) \cdot x$ & $\begin{array}{l}y(\mathrm{x})=45 \cdot \mathrm{x}, \text { em trinta dias, } \mathrm{x}= \\
30, \text { gasta-se } 1350 \text { litros }\end{array}$ \\
\hline 9 & 1 & 6 & $e=4$ & $y(x)=(9 \cdot 1+(9-4) \cdot 6) \cdot x$ & $\begin{array}{l}y(\mathrm{x})=39 \cdot \mathrm{x}, \text { em trinta dias, } \mathrm{x}= \\
30, \text { gasta-se } 1170 \text { litros }\end{array}$ \\
\hline 9 & 1 & 6 & $e=5$ & $y(x)=(9 \cdot 1+(9-5) \cdot 6) \cdot x$ & $\begin{array}{l}y(\mathrm{x})=33 \cdot \mathrm{x}, \text { em trinta dias, } \mathrm{x}= \\
\text { 30, gasta-se } 990 \text { litros }\end{array}$ \\
\hline 9 & 1 & 6 & $e=6$ & $y(x)=(9 \cdot 1+(9-6) \cdot 6) \cdot x$ & $\begin{array}{l}y(x)=27 \cdot x, \text { em trinta dias, } x= \\
\text { 30, gasta-se } 810 \text { litros }\end{array}$ \\
\hline 9 & 1 & 6 & $e=7$ & $y(x)=(9 \cdot 1+(9-7) \cdot 6) \cdot x$ & $\begin{array}{l}y(\mathrm{x})=21 \cdot \mathrm{x}, \text { em trinta dias, } \mathrm{x}= \\
\text { 30, gasta-se } 630 \text { litros }\end{array}$ \\
\hline 9 & 1 & 6 & $e=8$ & $y(x)=(9 \cdot 1+(9-8) \cdot 6) \cdot x$ & $\begin{array}{l}y(x)=15 \cdot x, \text { em trinta dias, } x= \\
30, \text { gasta-se } 450 \text { litros }\end{array}$ \\
\hline
\end{tabular}

Fonte: elaboração dos autores

Nesse modelo, que expressa a economia de água ao limpar os dejetos de uma bacia sanitária, estabelecemos que o número $e$ pertence ao conjunto dos números reais tal que $e$ seja 
um número qualquer entre zero e o volume de água armazenado na caixa acoplada ( $e \in \mathbb{R} \mid 0 \leq$ $e<\mathrm{V}_{\text {água }}$ ). Quando $e=0$ estamos considerando volumes de água iguais para limpar ambos os dejetos, já quando $e \neq 0$ há a distinção entre volume de água consumida para limpar dejetos sólidos e líquidos. Esse quadro apresenta os modelos de economia de água ao limpar dejetos sólidos e líquidos com diferentes volumes de água. Essa distinção se deve ao fata de quando que a construção de uma Descarga Digital permitisse digitar o volume de água para limpeza de dejetos líquidos da bacia sanitária. Além disso, o grupo "Natureza Blue” comparou os referidos resultados com o valor do volume de água que a norma 15.097/04 da ABNT estabelece para descarga de aparelhos sanitários com caixa acoplada, nesse caso, $\mathrm{V}_{\text {água }}=6 \mathrm{l}$. É importante ressaltar que nessa normativa não há distinção entre o volume de água consumido para limpar dejetos sólidos de dejetos líquidos, assim, $e=0$. Dessa forma se $\overline{\mathrm{x}}_{S}=1$ e $\overline{\mathrm{x}}_{L}=6$, temos:

$$
\begin{gathered}
y(\mathrm{x})=\left(\mathrm{V}_{\text {água }} \cdot \overline{\mathrm{x}}_{S}+\left(\mathrm{V}_{\text {água }}-e\right) \cdot \overline{\mathrm{x}}_{L}\right) \cdot \mathrm{x} \\
y(\mathrm{x})=(6 \cdot 1+(6-0) \cdot 6) \cdot \mathrm{x} \\
y(\mathrm{x})=42 \cdot \mathrm{x}
\end{gathered}
$$

Desse ponto de vista em trinta dias, $\mathrm{x}=30$, gasta-se 1260 litros, nos permitindo afirmar que mesmo que a caixa acoplada não atenda a norma 15.097/04 da ABNT, afinal a nossa gasta $9 l$ em vez de $6 l$, a construção de uma Descarga Digital favorece o cumprimento de tal normativa, pois possibilita a variação do volume de água usado para limpar a bacia sanitária como mostra o Quadro 1. Para auxiliar na compreensão, em uma planilha eletrônica, foi decidido criar um gráfico com as dez funções, Figura 9.

Figura 9 - Consumo de água para limpar os dejetos sólidos e líquidos



Fonte: Alves (2017)

Ao observamos o gráfico de cima para baixo, a primeira função refere-se ao consumo de água sem distinção para limpeza de dejetos sólidos e líquidos. Nela não há economia, $e=0$. ISSN 2526-2882 
Mas nas demais funções há, para calcularmos a economia de água ao final de um mês o grupo "Natureza Blue" fez a diferença entre o valor consumido da primeira função com as demais, ficou assim:

Quadro 2 - A economia de água

\begin{tabular}{|c|c|c|c|}
\hline$y_{e}(x)$ & $\boldsymbol{y}(\mathbf{x})$ quando $=\boldsymbol{o}$ & $\boldsymbol{y}(\mathbf{x})$ quando $e \neq 0$ & Economia \\
\hline$y_{e}(30)$ & $(9 \cdot 1+(9-0) \cdot 6) \cdot 30$ & $(9 \cdot 1+(9-1) \cdot 6) \cdot 30$ & $1890-1710=180 l$, se $e=1$ \\
\hline$y_{e}(30)$ & $(9 \cdot 1+(9-0) \cdot 6) \cdot 30$ & $(9 \cdot 1+(9-2) \cdot 6) \cdot 30$ & $1890-1530=360 l$, se $e=2$ \\
\hline$y_{e}(30)$ & $(9 \cdot 1+(9-0) \cdot 6) \cdot 30$ & $(9 \cdot 1+(9-3) \cdot 6) \cdot 30$ & $1890-1350=540 l$, se $e=3$ \\
\hline$y_{e}(30)$ & $(9 \cdot 1+(9-0) \cdot 6) \cdot 30$ & $(9 \cdot 1+(9-4) \cdot 6) \cdot 30$ & $1890-1170=720 l$, se $e=4$ \\
\hline$y_{e}(30)$ & $(9 \cdot 1+(9-0) \cdot 6) \cdot 30$ & $(9 \cdot 1+(9-5) \cdot 6) \cdot 30$ & $1890-990=900 l$, se $e=5$ \\
\hline$y_{e}(30)$ & $(9 \cdot 1+(9-0) \cdot 6) \cdot 30$ & $(9 \cdot 1+(9-6) \cdot 6) \cdot 30$ & $1890-810=1080 l$, se $e=6$ \\
\hline$y_{e}(30)$ & $(9 \cdot 1+(9-0) \cdot 6) \cdot 30$ & $(9 \cdot 1+(9-7) \cdot 6) \cdot 30$ & $1890-630=1260 l$, se $e=7$ \\
\hline$y_{e}(30)$ & $(9 \cdot 1+(9-0) \cdot 6) \cdot 30$ & $(9 \cdot 1+(9-8) \cdot 6) \cdot 30$ & $1890-450=1440 l$, se $e=8$ \\
\hline
\end{tabular}

Fonte: dos autores

Em que $y_{e}$ é a economia de água obtida em um mês ao diminuirmos a quantidade de água gasta para limpeza de dejetos líquidos na bacia sanitária. Contudo se os fabricantes de vasos sanitários respeitassem a 15.097/04 da ABNT, sem fazer nada o usuário já teria uma economia de 630 litros de água por mês, pois mesmo com e $=0$ teríamos:

$$
y_{e}(30)=[(9 \cdot 1+(9-0) \cdot 6) \cdot 30]-[(6 \cdot 1+(6-0) \cdot 6) \cdot 30]=1890-1260=630 l
$$

A partir desse gráfico das funções foi possível concluir que ao reduzir o consumo de água para limpeza dos dejetos líquidos além da economia de água, é possível alcançar os valores de consumo de água estabelecidos pela norma 15.097/04 da ABNT. Como vimos, para obter a economia fizemos a diferença entre o valor consumido em trinta dias da primeira função $(y(x)=63 \cdot x)$ com as demais. Usaremos a mesma ideia para encontrar a função economia $\left(y_{e}(\mathrm{x})\right)$, ou seja, vamos subtrair a função que considera o mesmo volume $(e=0)$ de água para limpar os dejetos da função que considera diferente o volume de água $(e \neq 0)$. Dessa forma temos:

$$
y_{e}(\mathrm{x})=y_{D S=D L}(\mathrm{x})-y_{D S \neq D L}(\mathrm{x}) \leftrightarrow y_{e}(\mathrm{x})=\left(e \cdot \overline{\mathrm{x}}_{L}\right) \cdot \mathrm{x} .
$$

Em que $y_{e}(\mathrm{x})$ é a economia de água; $e$ é a economia de água que se quer obter ao limpar os dejetos líquidos; $\overline{\mathrm{x}}_{L}$ é a média diária de dejetos líquidos e x é o tempo medido em dias. Por exemplo, queremos obter 5 litros de economia de água por descarga, $e=5$, em um mês com a nossa média de $\overline{\mathrm{x}}_{L}=6$, logo a economia será de: 


$$
y_{e}(\mathrm{x})=\left(e \cdot \overline{\mathrm{x}}_{L}\right) \cdot \mathrm{x}=5 \cdot 6 \cdot 30=900 l .
$$

Esse exemplo mostra que, com a Descarga Digital, o usuário obteria uma economia de 900 litros de água em trinta dias. Sabendo que criar uma tecnologia gerava economia, então o grupo "Natureza Blue" criou uma descarga em que pudesse digitar valores de um a nove litros, protótipo que é mostrado na Figura 10 - Protótipo da Descarga Digital.

Figura 10 - Protótipo da Descarga Digital

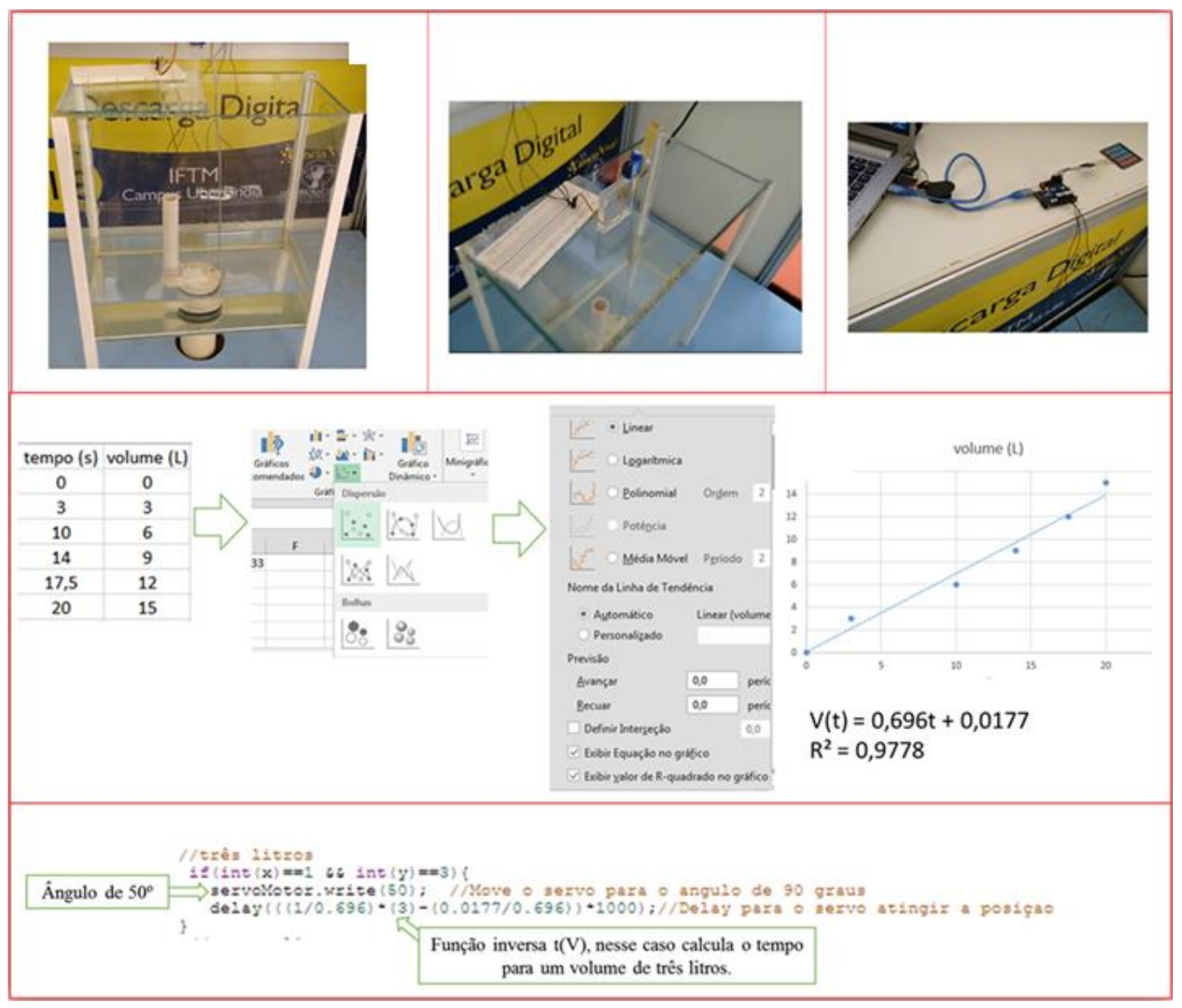

Fonte: Alves (2017)

Esse protótipo, que é um remixe dos saberes que os alunos adquiriram pela internet tiveram discussões e reflexões provocados por esse ambiente sociocultural digital entrelaçado ao analógico que gerou interações dos sujeitos com sujeitos e mediado por objetos, criando cenários investigativos de possibilidades de construção material e de saberes matemáticos, em que a práxis criadora (imaginação criadora mais ação criadora) impulsiona a criação que penetra "com sua criação a vida pessoal e social, especulativa e prática em todas as suas formas" (VIGOTSKI, 2009, p. 59). Essa práxis criadora só foi possível, pois no processo de 
produção do protótipo o grupo "Natureza Azul” construiu uma rede de informação e comunicação que apresentamos a seguir.

\section{Considerações Finais}

De acordo com dados da ANA (2019), compreendemos que a relevância do tema se mostrou capaz de fazer com que as pessoas envolvidas nessas ações buscassem soluções para o problema, por meio da modelagem matemática e de sua ligação com a cultura digital na educação matemática. Com este trabalho, além dos objetivos da pesquisa, estamos convictos de que o desenvolvimento de projetos com a modelagem matemática oferece importantes benefícios ao processo de ensino e aprendizagem. Porém, isso não nos deixa acreditar que a modelagem matemática, por si só, resolva os problemas e sane as dificuldades vivenciadas pela educação brasileira.

Educar, com a Matemática, estudantes da educação básica na realidade de um curso técnico em Meio Ambiente com tecnologias digitais da informação e comunicação, mostrou-se um desafio criativo e muito produtivo para esta pesquisa. O trabalho do grupo "Natureza Blue" abriu um campo de possibilidades educativas e de pesquisa na área da Educação Matemática com a Modelagem Matemática no contexto da Cultura Digital.

As experiências educativas com a Modelagem Matemática lendo o mundo do Arduino, para criar tecnologias de economia de água, foi cansativo, envolvente, divertido e recompensador. Uma implantação que entrelaçou Modelagem Matemática e Tecnologia em um processo de tornar o sujeito autor de conhecimento e tecnologia. Isso vai além de afetar nossa forma de ver as coisas do mundo, ela abre possibilidades para se criar desde máquinas automatizadas aos robôs, de acordo com nossa motivação e vontade. É contribuiu para a constituição de saberes oriundos da Modelagem Matemática e de sua ligação com a Cultura Digital na educação matemática. Ligação essa que nos propiciou superar os obstáculos inerentes ao próprio processo de construção dos saberes matemáticos, bem como acelerar o processo de apropriação desses saberes.

\section{Referências}

ABNT Associação Brasileira de Normas Técnicas. NBR 15097: Aparelho sanitário de material cerâmico: Requisitos e métodos de ensaio. Rio de Janeiro: Abnt, 2004. 37 p. Disponível em: <https://goo.gl/WkHWMC>. Acesso em: 24 abr. 2015.

ALVES, D. B. Modelagem Matemática no Contexto da Cultura Digital: uma Perspectiva de Educar pela Pesquisa no Curso de Técnico em Meio Ambiente Integrado ao Ensino Médio. 2017. 281 f. Tese (Doutorado) - Curso de Programa de Pós-graduação em Educação, Universidade Federal de Uberlândia, Uberlândia, 2017. Cap. 6. 
ANA - Agência Nacional de Águas. Uso eficiente da água traz ganho econômico e ambiental. 2019. Disponível em: <https://www.ana.gov.br/noticias-antigas/usoeficiente-da-a-gua-traz-ganho-econamico-e.2019-03-15.5033019754>. Acesso em: 12 mar. 2019.

BIEMbengut, Maria Salett. Modelagem Matemática no Ensino Fundamental. Blumenau: Edifurb, 2014.

CALDEIRA, A. D.; MEYER, J. F. da C. de A. Educação Matemática e Ambiental: uma proposta de formação continuada - e de mudanças. Zetetiké - CEMPEM - FE/UNICAMP, v. 9, n. 15-16, p. 155-170, jan./dez 2001.

CENSI (Blumenau). Kit Completo para Caixas Acopladas. 2019. Disponível em: <http://abre.ai/aizH>. Acesso em: 21 ago. 2019.

DEUZE, Mark. Participation, Remediation, Bricolage: Considering Principal Components of a Digital Culture. The Information Society. Bloomington, Indiana, p. 63-75, 10 dez. 2005.

FREIRE, P. Ação cultural: Para a liberdade e outros escritos. São Paulo: Paz e Terra, 2002.

GAZETA. Água: banheiro é o campeão de gasto. 2010. Disponível em: <https://goo.gl/e4N8hJ>. Acesso em: 04 mar. 2015.

IFTM- INSTITUTO FEDERAL DE EDUCAÇÃO, CIÊNCIA E TECNOLOGIA DO TRIÂNGULO MINEIRO. Projeto pedagógico do curso técnico em meio ambiente integrado ao ensino médio. 2013. Disponível em: <http://goo.gl/zxh4KE>. Acesso em: o8 mar. 2015 .

INMETRO - Instituto Nacional de Metrologia, Qualidade e Tecnologia. Sistema de Descarga: Relatório Final. 2014. Disponível em: <https://goo.gl/iEiWtU>. Acesso em: 17 out. 2015 .

MCTIC - MINISTÉRIO DA CIÊNCIA, TECNOLOGIA E INOVAÇÃO (Brasil). Semana Nacional de Ciência e Tecnologia - SNCT. 2015. Disponível em: < http://goo.gl/uvzNac >. Acesso em: 08 out. 2015.

MEYER, J. F. da C. de A.; CALDEIRA, A. D.; MALHEIROS, A. P. dos S.. Modelagem em Educação Matemática. Belo Horizonte: Autêntica, 2011.

O'SUllivaN, D.; IGOE, T. Pysical Computing: sensing and controlling the physical word with computers. Boston, Ma: Thomson Course Technology, 2004. 495 p.

RIPARDO, R. B.; OLIVEIRA, M. S.; SILVA, F. H. Modelagem Matemática e Pedagogia de Projetos: aspectos comuns. Alexandria: Revista de Educação em Ciência e Tecnologia, v.2, n.2, p.87-116, jul. 2009.

SKOVSMOSE, Ole. Cenários para investigação. Bolema: Boletim de Educação Matemática, Rio Claro, v. 1, n. 14, p. 1-22, 2 fev. 2015. Disponível em: <http://goo.gl/epsg4k>. Acesso em: 5 mar. 2015 . 
SMOLE, Kátia Cristina Stocco; DINIZ, Maria Ignez. Matemática: Ensino Médio - Vol. 1. São Paulo: Saraiva, 2013.

STEWART, J. Cálculo. São Paulo: Cengage Learning, 2015. V. 1.

UFRGS. Semelhança de Triângulos. 2016. Disponível em: <https://goo.gl/43yhx2>. Acesso em: 21 jan. 2016.

VIGOTSKI, L. S. Imaginação e criação na infância. São Paulo: Ática, 2009.

\section{Biografia Resumida}

Arlindo José de Souza Junior: Realizou o curso de graduação de Licenciatura em Matemática pela Universidade Estadual Paulista Júlio de Mesquita Filho (UNESP - Campus São José do Rio Preto); mestrado em Educação Matemática pela Universidade Estadual Paulista Júlio de Mesquita Filho (UNESP - Campus Rio Claro) e doutorado em Educação pela Universidade Estadual de Campinas (UNICAMP). Atualmente é professor titular da Universidade Federal de Uberlândia (UFU - Campus Uberlândia). Tem experiência na área de Educação, com ênfase em Educação Matemática, atuando principalmente nos seguintes temas: saberes docentes, educação popular e cultura digital.

Lattes: http://lattes.cnpq.br/9047733954063404

Contato: arlindoufu@gmail.com

Deive Barbosa Alves: Graduado em licenciatura em Matemática pela Universidade Federal de Uberlândia (2005), mestrado em Educação pela Universidade Federal de Uberlândia (2012) e doutorado na linha de pesquisa Educação em Ciências e Matemática pela mesma Universidade (2017). Atualmente participa do Núcleo de Pesquisa em Mídias na Educação (NUPEME) e do grupo de trabalho em Modelagem Matemática para a Educação Matemática da Sociedade Brasileira de Educação Matemática (GT1o - Modelagem Matemática). Professor e pesquisador do Programa de Pós-graduação em Ensino de Ciências e Matemática (PPGecim), Câmpus de Araguaína. Tem experiência na área de Educação Matemática, com ênfase em Modelagem Matemática no contexto da Cultura ISSN 2526-2882 
Digital, atuando principalmente nos seguintes temas: Modelagem Matemática para Educação Matemática, Educação pela Pesquisa, Educação Matemática na Cultura Digital, Objetos de Aprendizagem.

Lattes: http://lattes.cnpq.br/9350240158010161

Contato: deive@uft.edu.br 